\title{
Migration scenarii in extrasolar systems
}

\author{
A. Crida ${ }^{1}$ \\ ${ }^{1}$ Université de Nice Sophia-antipolis / Observatoire de la Côte d'Azur, boulevard \\ de l'Observatoire, BP4229, 06304 NICE cedex 4, France [crida@oca.eu]
}

\begin{abstract}
In this review talk, I present the theory of type I migration of small mass planets, and its latest developments that open the possibility of outward migration in the inner part of a protoplanetary disk. I also review the type II migration of giant planets, and mention the runaway, type III. Then, we focus on the migration of pairs of planets in resonance. The eccentricity of the planets raise, and possibly their mutual inclination as well. Also, the migration rate can be changed, and directed outward if the outer planet is the lighter. Last, we present a synthetic scenario of migration for the giant planets of our Solar System.
\end{abstract}

\section{Introduction}

Planets form in gaseous protoplanetary disks. The giant planet, in particular, acquire their hydrogen and helium envelope while the gas is still present. Therefore, during planetary formation, there has to be some planet-disk interactions. The planetary bodies are massive enough to perturb the disk density structure. This results in angular momentum exchange between the planet and the disk. This makes the orbit of a planet around the host star change. This phenomenon is know as planetary migration.

In this review, I will describe these planet disk interactions, and summarize all the possible migration scenarii, depending on the masses of the planet(s), on the parameters of the disk, and so on. In section 2., small mass planets are considered. The case of a locally isothermal disk is studied in subsection 2.1, while the case of adiabatic or radiative disks is discussed in subsection 2.2. In subsection 2.3, the role of turbulent fluctuations in the disk density is explained. In section 3., the case of giant planets is presented. Section 4 . is devoted to the more interesting case of pairs of planets. The possibility for resonance capture is discussed, and the consequences of the resonance on the evolution of the orbital elements of the planets is given. Last, in section 5., we draw a possible migration scenario for the outer solar system.

\section{Migration of a single small mass planet : type I migration}

A small mass planet on a circular orbit in a protoplanetary disk perturbs the disk density. The perturbation takes the form of a one-armed spiral wave, as can be seen in Figure 1. This wake exerts a force on the planet.

\subsection{Locally isothermal disk}

This is an Open Access article distributed under the terms of the Creative Commons Attribution-Noncommercial License 3.0, which permits unrestricted use, distribution, and reproduction in any noncommercial medium, provided the original work is properly cited. 

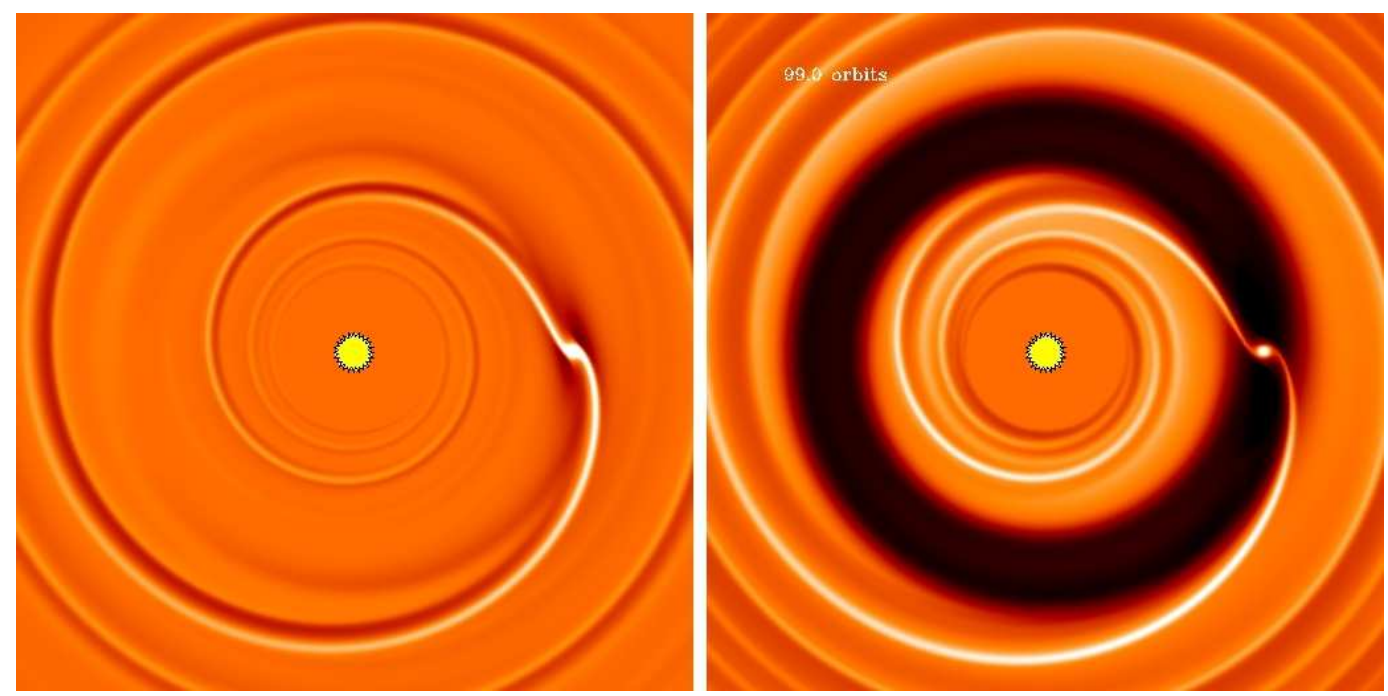

Figure 1.: Gas surface density of a disk perturbed by a planet. The star is at the center of the plot, the planet is on the right, in the middle of the white blob. Light color corresponds to over-density, dark corresponds to under-density. Left: Wake launched by a terrestrial planet in a protoplanetary disk. Right: gap opened by a giant planet. Images made with FARGO by F. Masset, modified by A. Crida.

\section{Differential Lindblad Torque}

The perturbation of the planet on the disk can be decomposed in Fourier series along the azimuth, which makes Lindblad resonances appear in the algebra. Goldreich \& Tremaine (1979) computed analytically the torque associated to a Lindblad resonance. The total torque exerted by all the outer (resp. inner) Lindblad resonances on the planet is negative (resp. positive) and called the one sided Lindblad torque.

Ward (1986) found that the torque at an inner and an outer resonance are not exactly opposite. Therefore, the total torque felt by the planet is not zero, but negative. It is called the differential Lindblad torque. Tanaka, Takeuchi, \& Ward (2002) provide a refined estimate of the differential Lindblad torque:

$$
\Gamma_{\mathrm{dLt}}=-(3.2+1.468 \zeta) \Gamma_{0}
$$

where $\zeta=\mathrm{d}(\log \Sigma) / \mathrm{d}(\log r)$, and

$$
\Gamma_{0}=\left(\frac{M_{p}}{M_{*}}\right)^{2} \Sigma r_{p}^{4} \Omega_{p} 2\left(\frac{H}{r_{p}}\right)^{-2} .
$$

with $M_{p}$ and $M_{*}$ the masses of the planet and the star respectively, $\Sigma$ the surface density of the gas disk, $r_{p}$ and $\Omega_{p}$ the orbital radius and angular velocity of the planet, and $H$ the thickness of the disk.

It appears that the planets lose orbital angular momentum, at a rate proportional to their masses squared. Consequently, their orbits shrink, they migrate inward, at a speed proportional to their mass. This is type I migration.

In a standard protoplanetary disk, an Earth-mass planet initially at 1 AU has a typical migration time of $2 \times 10^{5}$ years. This is much shorter than the lifetime of the disk. Our scenario number one is rather pessimistic: 
Scenario 1 : The planets are lost into their host star as soon as they form.

This was more or less our understanding of type I migration 5 years ago (back in 2005), which is obviously in contradiction with the simple fact that planets exist.

\section{Corotation torque}

However, not only the inner and the outer disk should be considered, but there is also a region between them in which the gas librates, on horseshoe-shaped orbits in the frame corotation with the planet (well-known in the restricted 3-body problem, see Fig. 2).

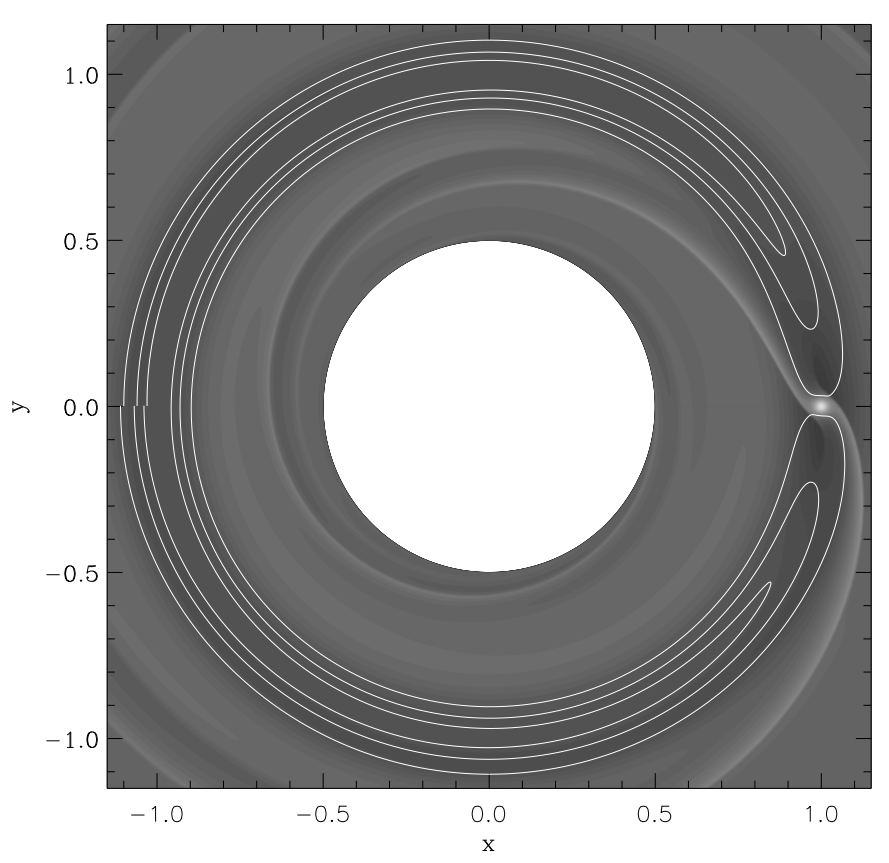

Figure 2.: Horseshoe orbits in the neighborhood of the planetary orbit, as seen from the frame corotating with the planet. Figure by F. Masset.

If an external mechanism maintains a density gradient (or more exactly, a vortensity gradient) in this region, then there can be a permanent asymmetry between the material making a U-turn inwards, and the material making a U-turn outwards. This creates on the planet a corotation torque. The corotation torque has been studied, for instance, by Ward (1991), Masset (2001), Casoli \& Masset (2009), Paardekooper \& Papaloizou (2009). In particular, Masset et al. (2006) have shown that at a locally strong positive vortensity gradient, the corotation torque is positive and larger than the negative differential Lindblad torque. Any migrating planet is thus trapped there. This planet trap could be found at the base of a disk jet, or the inner edge of a dead zone...

Scenario 2 : A planet is caught at a planet trap, and holds others by direct or indirect interactions. 


\subsection{Radiative disk}

More recently, Paardekooper \& Mellema (2006), Baruteau \& Masset (2008), Masset \& Casoli (2009) showed that in an adiabatic disk, entropy advection at the U-turns leads to the formation of a hotter, lighter plume behind the planet, and a colder, denser one in front of the planet. This makes a positive corotation torque, larger than the differential Lindblad torque. If the entropy profile is restored over a libration period of the horseshoe orbits, the planets should migrate outward (e.g. Kley \& Crida 2008). Paardekooper et al. (2010) showed that the total unsaturated torque is:

$$
\Gamma_{\text {tot }}=-(0.9+\zeta+1.7 \beta-7.9 \xi / \gamma) \Gamma_{0} / \gamma
$$

where $\beta=\mathrm{d}(\log T) / \mathrm{d}(\log r), \gamma$ is the adiabatic index, and $\xi=\beta-(\gamma-1) \zeta$.

This holds in the regions where the cooling time of the gas is larger than the time needed to make a U-turn in a horseshoe orbit. That is, in the innermost regions of the disk. This question of the saturation of the corotation torque is addressed by Paardekooper, Baruteau, \& Kley (2010).

Scenario 3 : Inward migration in the outer, optically thin regions ; outward migration in the inner, optically thick regions. A stable point appears, which location depends on the disk structure.

\subsection{Stochastic (type IV) migration}

Protoplanetary disks are expected to be turbulent. The turbulence creates random density variations, that in turn exert on the planet random torques. Nelson \& Papaloizou (2003) showed that the latter can dominate the differential Lindblad torque and the corotation torque, forcing the planet to follow a random walk instead of a monotonic migration; this regime is sometimes called type $I V$ migration.

Scenario 4: Random walk of the planets.

Adams \& Bloch (2009) showed that the ratio between the timescale for the diffusion of planets through random walk and the migration of planets through the above studied torques scales as $\Sigma H^{2} / M_{p}$. Therefore, diffusion dominates in the outer disk, while standard type I migration (scenario 3) should dominate in the inner regions.

\section{Migration of a single giant planet : type II migration}

The one sided Lindblad torque being proportional to the square of the planet mass (see Sect. 2.1), the one exerted by giant planets overcomes the viscous and pressure stresses internal to the gas disk. Thus, a giant planet perturbs the gas density profile and opens a gap in the protoplanetary disk (Lin \& Papaloizou 1986a; Crida, Morbidelli, \& Masset 2006). Then, the planet is locked into the gap, and can't migrate with respect to the gas disk. In contrary, it has to follow the viscous evolution of the disk, which is generally accretion onto the star. The planet migrates inwards at a viscous rate: this is type II migration (Lin \& Papaloizou 1986b).

Scenario 5: Type II migration of the giant planets. Slow decay toward the star, at the viscous timescale. 
This standard type II migration works as long as the mass of the planet is not too large with respect to the disk. Otherwise (in the late phases of the disk, or for very massive planets), the outer disk isn't able to push the planet at the viscous speed. The planet migrates more slowly, and as the inner disk accretes onto the star, a cavity opens (Crida \& Morbidelli 2007).

The second condition for pure type II migration is that the gap must be very clean. If not, there can be gas passing through, and exerting a corotation torque on the planet, that then decouples from the disk evolution (Crida \& Morbidelli 2007).

Under some conditions, the gas passing through the gap and exerting on the planet a corotation torque can lead to a runaway process : the faster the planet migrates, the more gas passes through the gap, and the faster the planet can migrate. This is type III migration (Masset \& Papaloizou 2003).

Scenario 6 : Type III, runaway migration. Inward or outward, in a few dozens of orbits.

\section{Migration of a pair of planets}

When two planets migrate in the same disk, they may have a convergent evolution of their semi-major axes. In that case, the ratio of their periods evolve, and can cross a commensurability. Then, the planets may lock in mean motion resonance. How the turbulent torques can destroy the resonance has been discussed by Adams, Laughlin, \& Bloch (2008), and Rein \& Papaloizou (2009).

Depending on the planets masses and the disk density, many kinds of resonance captures are possible. Pierens \& Nelson (2008) showed that the 3:2 is the most likely outcome for a Jupiter-Saturn pair. Note that Trojan, 1:1 configurations have also been observed by Thommes (2005), Beaugé et al. (2007), Cresswell \& Nelson (2009), and Crida (2009).

\subsection{Eccentricity and inclination evolution}

When the planets are trapped in mean motion resonance, their together migration leads to an increase of the eccentricities, for energy and angular momentum conservation reasons.

Then, the system can become unstable, the planets may have sooner or later (after the disk dispersal) a close encounter and one can be ejected, leaving the survival on a very eccentric orbit. Jurić \& Tremaine (2008) advocate that this could explain the eccentricity distribution of exoplanets (see also Raymond, Armitage, \& Gorelick 2009).

Scenario 7 : Convergent migration, resonance capture, eccentricity increase, instability and ejection of a planet.

But some resonant exoplanetary systems are stable, with reasonable $e$. Actually, the gas disk also tends to circularize the orbits of the planets. In particular, if the inner disk is properly taken into account, the planets can reach an equilibrium eccentricity, that can be low enough to ensure the stability of the system (Crida, Sándor, \& Kley 2008). This has probably been the case for instance in the system GJ876.

Scenario 8 : Convergent migration, resonance capture, eccentricity increase + damping, stable configuration. 


\section{EPJ Web of Conferences}

\subsection{Outward migration}

When the two planets in resonance are in a common gap, and the inner planet is more massive than the outer planet, then the pair of planet feels a total positive torque: the inner disk exerts a positive torque on the inner planet, which is larger in absolute value than the negative torque that the outer disk exerts on the smaller outer planet. Thus, the two planets migrate together outward (Masset \& Snellgrove 2001).

Scenario 9 : Outward (or slow) migration of a pair of planets in resonance.

Morbidelli \& Crida (2007) studied how the migration rate depends on the disk parameters in the case of a Jupiter-Saturn pair. Crida, Masset, \& Morbidelli (2009) showed that the migration can go outward on the long run, up to where the outer disk gets thick (or viscous) enough that the gap opened by the outer planet get significantly filled. This could explain the recently directly observed exoplanets, around Fomalhaut (Kalas et al. 2008) or HR8799 (Marois et al. 2008).

\section{Case of the Solar System}

From what we have seen above, we can draw a consistent scenario for the solar system. We know that Jupiter and Saturn should lock in 3:2 mean motion resonance. Due to their mass ratio, they should then have a slow inward or outward migration (if not almost stationary), that prevents Jupiter from falling into the Sun. Actually, it has been proposed after the OHP-2010 conference that Jupiter first migrated inward in type II migration, down to $1.5 \mathrm{AU}$, and then got caught in 2:3 MMR by Saturn, and made a grand tack to migrate outward to $\sim 5$ AU (Walsh et al. 2010). This of course shapes the planetesimals and embryos disk in the terrestrial planets region. It helps forming a small Mars, and the Main Asteroid Belt.

Anyway, it is clear that Uranus and Neptune should then be stopped in their inward migration by Saturn, and caught in mean motion resonances by it. The exact resonance configuration depends on the disk parameters, but the four giants of the outer Solar System most likely end in a fully resonant configuration (Morbidelli et al. 2007). They avoid migration into the Sun thanks to the mass ratio between Jupiter and Saturn, if the disk parameters are not too extreme (Crida (2009)).

Then, once the gas disk disappears, this resonant configuration can be stable for hundreds of millions of years. But if it is slowly perturbed by a remnant disk of planetesimals beyond Neptune, at some point, the resonance can be broken. The system being very compact then runs unstable. A global instability takes place, in which the planetesimals disk is destroyed and the planetesimals are ejected into the inner solar system at an unusual high rate : this explains the Late Heavy Bombardment that took place 600 Myrs after the formation of the Solar System and left the basins on the Moon (Gomes et al. 2005). The orbits of the giant planets are also strongly modified, and they reach their present semi-major axes and eccentricities (Tsiganis et al. 2005). In addition, Jupiter and Neptune can capture their Trojan asteroids during this process (Morbidelli et al. 2005). The giant planets catch their irregular satellites when they have close encounter with each other while planetesimals are running all around (Nesvorný, Vokrouhlický, \& Morbidelli 2007). Finally, the planetesimals disk is depleted and shaped by the outward migration of Neptune, and becomes the Kuiper Belt (Levison et al. 2008). This scenario is often called the Nice-model. 


\section{Conclusion}

As we have seen, migration is an unavoidable process in the formation of planets inside a protoplanetary gaseous disk. Many scenarios are possible, offering almost all possibilities, but one shouldn't conclude that migration could for this reason be neglected. On the contrary, it is very important to know what the parameters of the problem are (viscosity, density, aspect ratio of the disk, mass of the planet, planet-planet interactions) to find which scenario applies. And conversely, the observed state of a planetary system can give us many indications on the conditions in which it must have been formed.

The consequences of latest discoveries on the type I migration rate on planetary migration have not yet been fully analyzed, and the migration of several planets interacting with each others is also a promising and expending field. The numerous discoveries of exoplanets push theorists towards a statistic approach of the formation of planetary systems : it is now impossible to build a model for every planets. Migration theories and their applications are still improving, and reaching maturity, and they will play their role in painting the global picture of planetary formation.

\section{References}

Adams, F. C., Laughlin, G., \& Bloch, A. 2008, ApJ, 683, 1117-1128

Adams, F. C. \& Bloch, A. M. 2009, 701, 1381-1397

Baruteau, C. \& Masset, F. 2008, ApJ, 672, 1054-1067

Beaugé, C., Sánodr, Zs., Érdi, B., \& Süli, Á. 2007, A\&A, 463, 359-367

Casoli, J. \& Masset F. 2009, 703, 845-856

Cresswell, P. \& Nelson, R. P. 2009, A\&A, 493, 1141-1147

Crida, A., Morbidelli, A., \& Masset, F. 2006, Icarus, 181, 587-604

Crida, A. \& Morbidellli, A. 2007, MNRAS, 377, 1324-1336

Crida, A., Sándor, Z., \& Kley, W. 2008, A\&A, 483, 325-337

Crida, A. 2009, ApJ, 698, 606-614

Crida, A., Masset, F. \& Morbidelli, A. 2009, ApJ, 705, L148-L152

Gomes, R., Levison, H. F., Tsiganis, K., \& Morbidelli, A. 2005, Nature , 435, 466-469

Goldreich, P. \& Tremaine, S. 1979, ApJ, 233, 857-871

Jurić, M. \& Tremaine, S. 2008, ApJ, 686, 603-620

Kalas, P., Graham, J. R., Chiang, E., Fitzgerald, M. P., Clampin, M., Kite, E. S., Stapelfeldt, K., Marois, C., \& Krist, J. 2008, Science, 322, 1345-

Kley, W. \& Crida, A. 2008, A\&A, 487, L9-L12

Levison, H. F., Morbidelli, A., Vanlaerhoven, C., Gomes, R., \& Tsiganis, K. 2008, Icarus, 196, 258-273

Lin, D. N. C. \& Papaloizou, J. 1986, ApJ, 307, 395-409

Lin, D. N. C. \& Papaloizou, J. 1986, ApJ, 309, 846-857

Marois, C., Macintosh, B., Barman, T., Zuckerman, B., Song, I., Patience, J., Lafrenière, D., \& Doyon, R. 2008, Science, 322, 1348-

Masset, F. S. 2001, ApJ, 558, 453-462

Masset, F. \& Snellgrove, M. 2001, MNRAS, 320, L55-L59

Masset, F. S. \& Papaloizou, J. C. B. 2003, ApJ, 588, 494-508

Masset, F. S., Morbidelli, A., Crida, A., \& Ferreira, J. 2006, ApJ, 642, 478-487 
Masset \& Casoli 2009, ApJ, 703, 857-876

Morbidelli, A., Levison, H. F., Tsiganis, K., \& Gomes, R. 2005, Nature , 435, 462-465

Morbidelli, A. \& Crida, A. 2007, Icarus, 191, 158-171

Morbidelli, A., Tsiganis, K., Crida, A., Levison, H. F., \& Gomes, R. 2007, AJ, 134, $1790-$

Nelson, R. P. \& Papaloizou, J. C. B. 2004, MNRAS, 350, 849-864

Nesvorný, D., Vokrouhlický, D., \& Morbidelli, A. 2007, AJ, 133, 1962

Paardekooper, S.-J. \& Mellema, G. 2006, A\&A, 459, L17-L20

Paardekooper, S.-J. \& Papaloizou, J. C. B. 2009, MNRAS, 394, 2283-2296

Paardekooper, S.-J., Baruteau, C., Crida, A., \& Kley, W. 2010, MNRAS, 401, 19501964

Paardekooper, S.-J., Baruteau, C., \& Kley, W. 2010, MNRAS, in press

Pierens, A. \& Nelson, R. P. 2008, A\&A, 482, 333-340

Raymond, S. N., Armitage, P. J., \& Gorelick, N. 2009, ApJ, 699, L88-L92

Rein, H. \& Papaloizou, J. C. B. 2009, A\&A, 497, 595-609

Tanaka, H., Takeuchi, T., \& Ward, W. R. 2002, ApJ, 565, 1257-1274

Thommes, E. W. 2005, ApJ, 626, 1033-1044

Tsiganis, K., Gomes, R., Morbidelli, A. \& Levison, H. F. 2005, Nature , 435, 459-461

Ward, W. R. 1991, in Lunar and Planetary Institute Conference Abstracts, 1463-1464

Walsh, K. J., Morbidelli, A., Raymond, S. N., O'Brien, D. P., Mandell, A. 2010, in Bulletin of the American Astronomical Society, \#42, 947-

Ward, W. R. 1986, Icarus, 67, 164-180 